\title{
KDM5B protein expressed in viable and fertile $\triangle$ ARID mice exhibit no demethylase activity
}

\author{
SHIRIN JAMSHIDI ${ }^{1}$, STEVEN CATCHPOLE $^{2,4}$, JIE CHEN $^{3}$, CHI WAI ERIC SO ${ }^{3}$, JOY BURCHELL ${ }^{2 *}$, \\ KHONDAKER MIRAZ RAHMAN $^{1 *}$ and JOYCE TAYLOR-PAPADIMITRIOU ${ }^{2 *}$
}

\begin{abstract}
${ }^{1}$ Institute of Pharmaceutical Science, School of Cancer and Pharmaceutical Sciences, King's College London, London SE1 9NN; ${ }^{2}$ Breast Cancer Biology, Innovation Hub, School of Cancer and Pharmaceutical Sciences, King's College London, Guy's Cancer Centre, Guy's Hospital, London SE1 9RT; ${ }^{3}$ Leukaemia and Stem Cell Biology Group, School of Cancer and Pharmaceutical Sciences, Denmark Hill Campus, King's College London, London SE5 9RJ, UK
\end{abstract}

Received May 23, 2021; Accepted August 9, 2021

DOI: $10.3892 / \mathrm{ijo} .2021 .5276$

\begin{abstract}
Post-translational modification of histones serve a crucial role in the control of gene transcription. Trimethylation of lysine 4 on histone 3 is associated with transcription activation. There are currently six known methylases and six known demethylases that can control the methylation status of this site. Lysine demethylase 5B (KDM5B) is one such demethylase, which can repress gene expression. In particular KDM5B has been found to be overexpressed in a number of cancer types, and small-molecular weight inhibitors of its demethylase activity have been identified. Previous characterisation of $K d m 5 b$ knock-out mice has revealed that this genotype leads to either embryonic or neonatal lethality. However, the $\Delta \mathrm{A}-\mathrm{T}$ rich interaction domain ( $\triangle$ ARID)-KDM5B strain of mice, which have the ARID domain and five amino acids within the Jumonji (Jmj)N domain spliced out from KDM5B, remain viable and fertile. In the present study, $\triangle$ ARID-KDM5B was found to have no demethylase activity as determined by in vitro demethylase assays and by immunofluorescence in transfected Cos-1 cells. Furthermore, molecular dynamic simulations revealed conformational changes within the $\triangle$ ARID-KDM5B structure compared with that in WT-KDM5B, particularly in the $\mathrm{JmjC}$ domain, which is responsible for the catalytic activity of
\end{abstract}

Correspondence to: Professor Joyce Taylor-Papadimitriou, Breast Cancer Biology, Innovation Hub, School of Cancer and Pharmaceutical Sciences, King's College London, Guy's Cancer Centre, Guy's Hospital, Great Maze Pond, London SE1 9RT, UK E-mail: joyce.taylor-papadimitriou@kcl.ac.uk

Present address: ${ }^{4}$ Department of Medical and Molecular Genetics, King's College London, Guy's Hospital, London SE1 9RT, UK

\section{${ }^{*}$ Contributed equally}

Key words: histone demethylases, lysine demethylase 5B, $\Delta \mathrm{A}-\mathrm{T}$ rich interaction domain mice, loss of demethylase activity, molecular dynamics simulation
WT-KDM5B. This supports the experimental data that shows the loss of demethylase activity. Since $K d m 5 b$ knock-out mice show varying degrees of lethality, these data suggest that KDM5B serves a crucial function in development in a manner that is independent of its demethylase activity.

\section{Introduction}

The H3K4me3 histone mark is frequently found at the promoters of genes that are undergoing active transcription $(1,2)$. In mammals, to methylate $\mathrm{H} 3 \mathrm{~K} 4$ there are six methyltransferases that can function as components of complexes (3), whilst there are six demethylases that can remove these methyl groups from this mark. Specifically, two of these demethylases, namely lysine-specific demethylase (LSD or KDM)1/KDM1A and LSD2/KDM1B, belong to the flavin adenine dinucleotide-dependent homologues of the amine oxidase family, which can remove methyl groups from dimethylated and monomethylated H3K4 (4). By contrast, four KDM5 proteins, KDM5A, KDM5B, KDM5C and KDM5D, are members of the Jumonji (Jmj)C class of Fe (II)- and 2-oxoglutarate-dependent proteins, which can demethylate $\mathrm{H} 3 \mathrm{~K} 4 \mathrm{me} 3$ and $\mathrm{H} 3 \mathrm{~K} 4 \mathrm{me} 2$ through the JmjC domain (5). Interacting with repressor complexes, the KDM1 and KDM5 demethylases can repress gene transcription and are overexpressed in a wide range of cancers, including renal cell carcinoma and head and neck cancers $(4,5)$. KDM5B (also known as JARID1B or PLU-1) was first identified as being upregulated in breast $(6,7)$ and prostate cancer (8). These two types of cancer have been the focus of previous studies in oncogenesis $(9,10)$.

The KDM5 family of demethylases are unique among those that use an $\mathrm{Fe}^{2+}$ - and 2-oxoglutarate-dependent mechanism for removing methyl groups $(5,6)$. The catalytic core of KDM5 is separated into the JmjN and JmjC domains by sequences, which include the A-T rich interaction domain (ARID or BRIGHT domain) and the plant homeodomain 1 (PHD1) $(5,6)$. The DNA-binding ARID domains in KDM5A and B (Fig. 1A) can bind to GC-rich sequences $(11,12)$, whilst the PHD1 domain interacts with unmethylated H3K4 (13). However in both KDM5A and B proteins, the JmjN and JmjC domains lie adjacent to each other and interact closely (14-16). 
Previous studies using the expression of mutated constructs found that deletions of either the ARID, JmjN or JmjC domains led to the loss of histone demethylase function $(7,8)$. However, linking the JmjN and JmjC domains of KDM5B, which results in the deletion of the ARID and PHD1 domains, did not decrease demethylase activity in vitro (17). In addition, a shortened construct of KDM5B, which contains deletion of the PHD1 domain and most of the ARID domain (amino acids 102-369 deleted), retains its demethylase activity (14). However, although apparently dispensable in terms of demethylase activity, the PHD1 domain can influence the re-modelling of the catalytic core by binding to $\mathrm{H} 3 \mathrm{~K} 4 \mathrm{me} 0(15,18)$.

Human and mouse KDM5B share a $95 \%$ sequence homology at protein level (6). To investigate the function of $\mathrm{KDM}$ 5B, a number of knockout (KO) genotypes of mouse have been developed. The $K d m 5 b$ KO C57BL/6 mice developed by Albert et al (19), which targeted exon 6, resulted in neonatal lethality due to respiratory failure resulting from neurological abnormalities. Another homozygous $K d m 5 b \mathrm{KO}$ mice developed by Catchpole et al (20) generated on the same C57BL/6 background, which targeted exon 1, caused early embryonic lethality. In addition, a $K d m 5 b \mathrm{KO}$ mice developed by Zou et al (21), with a deletion between exon 2 and exon 3, remain viable and fertile on the C57BL/6 background. However, when bred on the FVB/N background, both males and females show increased rates of mortality and females showed reduced fertility and abnormal mammary gland development. It is possible that targeting different domains in KDM5B can explain different phenotypes (21).

A $K d m 5 b$ transgenic mouse model was previously developed (20), where a splicing event led to the removal of exons 2, 3 and 4 via the splicing of exon 1 to 5 . This mouse genotype is known as the $\triangle$ ARID mouse, which is viable and fertile, with the only phenotype being a transient delay in mammary gland development (20). In this mouse, in-frame splicing of the primary transcript from exons 1-5 resulted in the deletion of the entire ARID domain. However, this splicing event also resulted in the truncation of the JmjN domain, with amino acids Asp69, Trp70, Gln71, Pro72 and Pro73 deleted from the carboxyl end. However, all other domains, including the PHD1 and JmjC domains, remain intact (Fig. 1B).

Whilst the JmjN domain is clearly required for demethylase activity, neither the deletion nor the mutation studies aforementioned examined whether the partial deletion of the JmjN domain together with the ARID domain affects demethylase activity. Since the $\triangle$ ARID mice with this deletion remain viable and fertile (20), it is important to establish whether the KDM5B protein they express has demethylase activity. In the present study this question was addressed by developing a plasmid construct encoding $\triangle$ ARID-KDM5B and assaying demethylase activity in Cos-1 cells transfected with this construct. In silico homology modelling followed by molecular dynamics (MD) simulations was then applied to document any changes in the domains of $\triangle \mathrm{ARID}$-KDM5B, with the aim of providing supportive evidence for the loss of demethylase activity.

\section{Materials and methods}

Construction of the Kdm5b-AARID construct in the pcDNA3.1 plasmid. Mouse $W T-K d m 5 b$ cDNA was originally isolated from a mouse mammary cell line 410.4 (obtained from the originator, Dr Gloria Heppner, Michigan Cancer Foundation, Detroit, USA) by Madsen et al (22). To create the $K D M 5 B-\triangle A R I D$ construct, as previously seen in the $\triangle \mathrm{ARID}$ mice (20), the $K D M 5 B$ sequence corresponding to the splicing of exon 1 to exon 5 was designed by the authors and synthesised by GenScript for ligation into the pcDNA3.1 plasmid (cat. no. V87020; Thermo Fisher Scientific, Inc.), which was originally carrying the WT-KDM5B sequence but was cut out using NotI and Bsu36I. Fig. 1C shows the effect of this splicing event on the protein sequence.

Immunofluorescence staining. Mouse WT-Kdm5b, mouse $\triangle A R I D-K d m 5 b$ cDNA and a human $\triangle \mathrm{JmjC}$ construct were all encoded in the pcDNA3.1 plasmid (Sigma-Aldrich; Merck KGaA). They were transfected into Cos-1 cells using Lipofectamine ${ }^{\circledR} 2000$ (Invitrogen; Thermo Fisher Scientific, Inc.) according to the manufacturer's protocol. In total, $0.5 \mu \mathrm{g}$ plasmids were used per transfection. Cos-1 (cat. no. CRL-1650; American Type Culture Collection) were routinely cultured in DMEM (Thermo Fisher Scientific, Inc.) with $10 \%$ foetal calf serum (Thermo Fisher Scientific, Inc.), at $37^{\circ} \mathrm{C}$ and $5 \% \mathrm{CO}_{2}$, The plasmid containing KDM5B with a deletion in the JmjC domain, derived from the WT- $K d m 5 b$ cDNA, was provided by Dr Degui Chen, State Key Laboratory of Molecular Biology, Shanghai Institutes for Biological Sciences (Shanghai, China) (8). In total, $24 \mathrm{~h}$ after transfection the cells were fixed in $4 \%$ paraformaldehyde for $15 \mathrm{~min}$ at room temperature, permeabilised with Triton X-100 for $10 \mathrm{~min}$ at room temperature, washed and sequentially stained with an antibody to KDM5B at a dilution of 1:700 for $2 \mathrm{~h}$ at room temperature. This antibody was an in-house rabbit antiserum that was raised to the C-terminal domain of human KDM5B corresponding to amino acid residues 1283-1473 (expressed and produced in $E$ coli $M 15$ ). This antibody shows specificity for KDM5B and reacts with both human and mouse KDM5B (23). To identify H3K4me3 staining, a mouse monoclonal antibody (cat. no. ab1012; dilution, 1:100; Abcam) was used, with incubation for $2 \mathrm{~h}$ at room temperature. Binding of the primary antibodies was visualised using Alexa Fluor ${ }^{\circledR} 546$ donkey anti-rabbit IgG (cat. no. A10040) and Alexa Fluor ${ }^{\circledR} 488$ goat anti-mouse IgG (cat. no. A-10680; Molecular Probes; Thermo Fisher Scientific, Inc.) secondary antibodies, both diluted to 1:500 and incubation was for $1 \mathrm{~h}$ at room temperature. Cells were then mounted in mounting medium containing DAPI (cat. no. ab104139; Abcam) and visualized under a Zeiss AX10 immunofluorescence microscope (magnification, x630).

Histone demethylation analysis. Mouse $W T-K d m 5 b$ and mouse $\triangle A R I D-K d m 5 b$ cDNA both encoded in the pcDNA3.1 plasmid and the vector control (pcDNA3.1; Sigma-Aldrich; Merck KGaA), were transfected into 293T cells using Polyethylenimine (cat. no. 23966-2; Polysciences, Inc.) with $10 \mu \mathrm{g}$ plasmid used per transfection. 293T cells (ATCC; cat. no. CRL-3216) were routinely cultured in DMEM with $10 \%$ foetal calf serum, at $37^{\circ} \mathrm{C}$ and $5 \% \mathrm{CO}_{2}$. After $48 \mathrm{~h}$ cells were suspended in a hypotonic cell lysis buffer $(5 \mathrm{mM}$ HEPES, $50 \mathrm{mM} \mathrm{KCl}, 10 \mathrm{mM} \mathrm{MgSO} \cdot 7 \mathrm{H}_{2} \mathrm{O}, 0.05 \% \mathrm{NP}-40,3 \mathrm{mM}$ DTT, $1 \mathrm{mM}$ PMSF and a cocktail of protease inhibitors), incubated at room temperature for $1 \mathrm{~min}$ and centrifuged 
A

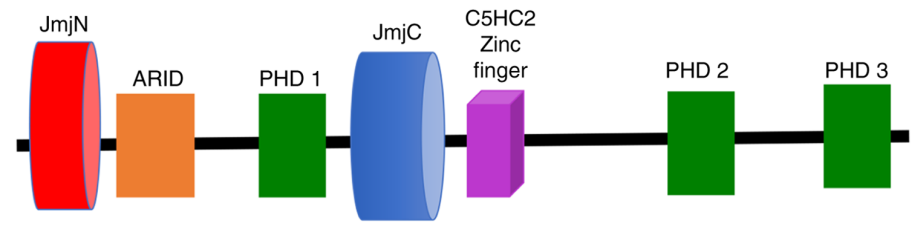

B
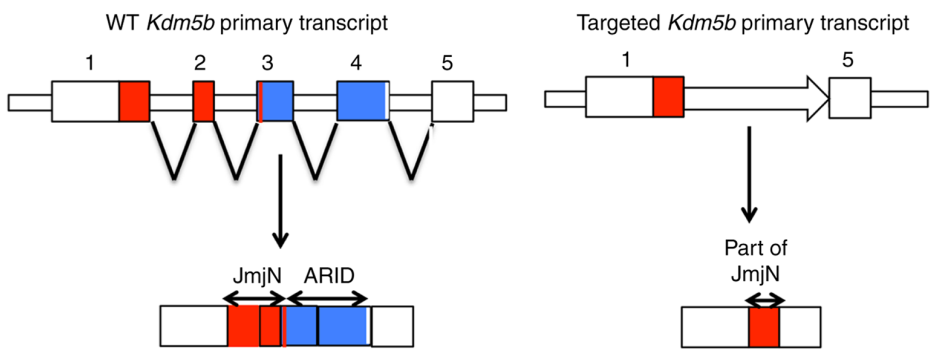

C

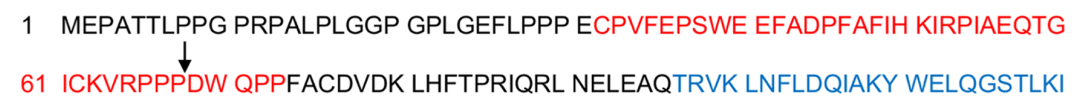

121 PHVERKILDL FQLNKLVAEE GGFAVVCKDR KWTKIATKMG FAPGKAVGSH IRGHYERILN

181 PYNLFLSGDS LRCLQKPNLT SDTKDKEYKP HDIPQRQSVQ PAETCPPARR AKRMRAEAMN

241 IKIEPEEATE ARTHNLRRRM GCTTPKWENE KEMKSTIKQE PTEKKDCELE SEKEKPKSRA

301 KKTATAVDLY VCLLCGSGND EDRLLLCDGC DDSYHTFCLV PPLHDVPKGD WRCPKCLAQE

361 CNKPQEAFGF EQAARDYTLR TFGEMADAFK SDYFNMPVHM VPTELVEKEF WRLVSTIEED

421 VTVEYGADIA SKEFGSGFPV RDGKIKISPE EEEYLDSGWN LNNMPVMEQS VLAHITADIC

Figure 1. $K d m 5 B$ RNA is spliced from exon 1 to exon 5 in the mouse $\triangle$ ARID- $K d m 5 B$ construct. (A) Linear domain structure of mouse KDM5B. (B) Schematic representation depicting the mRNA splicing event of the $W T-K d m 5 b$ primary transcript that results in the production of the $\Delta \mathrm{ARID}-K d m 5 B$ transcript. Left, red areas in exons 1, 2 and 3 represent the JmjN domain; blue areas in exons 3 and 4 represent the ARID domain. Right, the mRNA splicing event of the engineered $K d m 5 b$ primary transcript resulting in the splicing together of exons 1 and 5, which deletes the ARID domain and part of the JmjN domain. (C) WT murine KDM5B protein sequence showing the JmjN domain (red, residues 32-73), the ARID domain (blue, 97-187), and the beginning of the JmjC domain (green, 453-619). Arrows indicate the beginning and end of the spliced region observed in the $\triangle$ ARID-KDM5. Jmj, Jumonji; ARID, A-T rich interaction domain; PHD1, plant homeodomain 1; KDM5, lysine-specific demethylase 5B; WT, wild-type.

for $5 \mathrm{~min}$ at $1,000 \mathrm{x} \mathrm{g}$ at $4^{\circ} \mathrm{C}$. The cell pellet was then resuspended and washed three times in ice-cold RSB washing buffer $(10 \mathrm{mM} \mathrm{NaCl}, 10 \mathrm{mM}$ Tris- $\mathrm{HCl} \mathrm{pH} 8.0$ and $3 \mathrm{mM}$ $\mathrm{MgCl}_{2}$ ). Subsequently, $0.4 \%$ trypan blue staining was used to check for complete nuclear extraction as viewed under a light microscope. After nuclear extraction, intact nuclei were positive for trypan blue staining under the microscope and are smaller in size compared with those in control 293T cells. Intact cells with whole nuclei were stained negative.

Finally, nuclear proteins were extracted using RIPA buffer (50 mM Tris $\mathrm{HCl}, 150 \mathrm{mM} \mathrm{NaCl}, 1.0 \%$ (v/v) NP-40, $0.5 \%$ (w/v) sodium deoxycholate, $1.0 \mathrm{mM}$ EDTA, $0.1 \%(\mathrm{w} / \mathrm{v}) \mathrm{SDS}$ and $0.01 \%(\mathrm{w} / \mathrm{v})$ sodium azide, $\mathrm{pH}$ 7.4) with protease inhibitors. The nuclear extracts were incubated on ice for $10 \mathrm{~min}$ before being cleared by centrifugation at $100,000 \mathrm{x} \mathrm{g}$ for $10 \mathrm{~min}$ at $4^{\circ} \mathrm{C}$. Protein concentration was measured using Bradford Assay (Bio-Rad Laboratories, Inc.). The demethylation activity was measured using the histone $\mathrm{H} 3(\mathrm{~K} 4)$ demethylase activity quantification assay kit from Abcam (cat. no. ab113455) using $5 \mu \mathrm{g}$ each extract.

Statistical analysis. A two-tailed student's t-test was used to analyse the difference between the WT-KDM5B and $\triangle$ ARID-KDM5B results from the demethylase assay. Data are presented as the mean \pm standard deviation. $\mathrm{P} \leq 0.05$ was considered to indicate a statistically significant difference. The results presented represent three biological replicates.

Homology modelling. The Swiss-Model webserver (https://swissmodel.expasy.org) $(24,25)$ was used for the homology modelling of the mouse WT-KDM5B structural model using the FASTA-formatted target protein sequence with the UniProt entry number 'Q80Y84' (https://www. uniprot.org/uniprot/Q80Y84). The crystal structure of human WT-KDM5A with the PDB ID 5K4L was used as the template (16). The template and KDM5B shared a sequence identity of $64.43 \%$. The 3D structure of the KDM5A in the PDB format was without any gaps and all of the segments were solved. The mutant $\triangle$ ARID-KDM5B (missing amino acid residues 69-191; Fig. 1C) was generated by manipulating the primary file in a text editor. All systems were minimised and equilibrated using the AMBER version 16 software (https://ambermd.org/) (26) before performing the MD simulations. Evaluation of the homology model was performed by calculating the Z-score using AMBER 16. The Z-score is an estimation of the comparability of the model to the experimentally-derived structures with similar sizes of the target protein. The Z-score for the $\triangle \mathrm{ARID}$-KDM5B model was $0.68 \pm 0.05$. Z-scores $\sim 0.0$ would indicate a native-like structure, whilst Z-scores $<-4.0$ would indicate a low-quality model. Therefore, 
the $\mathrm{Z}$ score of the present model lied within the range of scores calculated for proteins of similar size with experimentally determined structures, indicating a good overall quality of the built model.

MD simulations. Topology and coordinate files for the WT-KDM5B and $\triangle$ ARID-KDM5B systems were generated in TIP3P water using AMBER16 (https://ambermd.org/) (26) with the 'tLEaP module' of AMBER 16 (26). The systems were minimised in two stages using the AMBER 16 package program. In the first step, 1,000 steps of minimisation with restraint on solvent were performed, which this was followed by 2,500 steps of minimisation without restraint. MD was performed in three stages using the AMBER program. First, 500-psec heating steps were accomplished from 0-300K with restraint on solvents. Subsequently, 500 ps equilibration steps in constant temperature was run before $100 \mathrm{~ns}$ sampling or production steps (NPT) finally completed the simulation.

Periodic boundary conditions were applied during the simulations. The NPT runs used the Langevin algorithm (https://ambermd.org/) (26), whilst the pressure was controlled using the isotropic position scaling protocol in the AMBER barostat. The Particle Mesh Ewald method was employed with a cut-off radius of $12 \AA$ for electrostatic and van der Waals interactions for proteins (27).

\section{Results}

Mouse $\triangle A R I D-K D M 5 B$ does not have demethylase activity. The deletion that occurred in the $\triangle$ ARID-KDM5B by splicing out exons 2-4 resulted in the expression of a smaller transcript and protein with the ARID domain (residues 96-188) and part of the JmjN domain deleted (residues 69-73; Fig. 1B). cDNAs encoding the WT- Kdm5b and the $\triangle$ ARID-Kdm $5 b$ sequence were transfected into Cos-1 cells, which do not endogenously express KDM5B (23). The cells were co-stained with antibodies for $\mathrm{H} 3 \mathrm{~K} 4 \mathrm{me} 3$ and KDM5B. A human $K D M 5 B$ construct with the JmjC domain deleted, which was known to lack demethylase activity $(7,8)$, was also transfected into Cos-1 cells. Cells expressing the $\triangle$ ARID-KDM5B protein showed no clear reduction in the level of H3K4me3, which was also observed in cells transfected with the catalytically-dead JmjC mutant construct (Fig. 2A). However, H3K4me3 was markedly downregulated in cells expressing WT-KDM5B. These data indicate that the deletion that occurred in murine $\triangle$ ARID-KDM5B results in the loss of demethylase activity.

To further confirm these findings, in vitro demethylase assays were performed on $293 \mathrm{~T}$ cells transfected with WT-Kdm $5 b, \Delta$ ARID-Kdm $5 b$ or vector control construct. The nuclear proteins were extracted and subjected to an ELISA-based demethylase activity assay. As shown in Fig. 2B, there was a significant loss of demethylase activity in the $\triangle$ ARID-KDM5B protein compared with that in the wild-type protein, with the $\triangle$ ARID-Kdm5b nuclear extract yielding similar results to the vector control. Together with the results from immunofluorescence staining, these data indicate that the loss of five amino acids from the JmjN, in addition to the ARID domain, results in the loss of enzymatic activity of KDM5B.
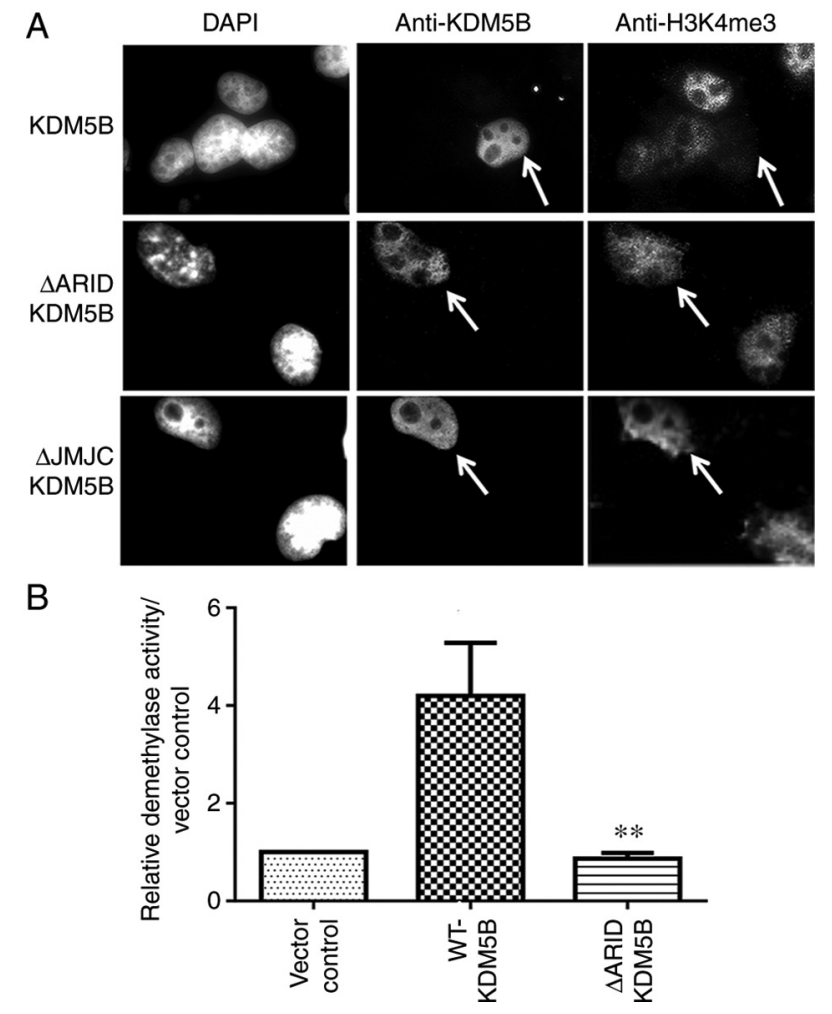

Figure 2. $\triangle$ ARID-KDM5B protein lacks demethylase activity. (A) Mouse $K d m 5 B$ cDNA was constructed with an exon 2 to 4 deletion to produce the $\triangle \mathrm{ARID}-K d m 5 B$ product before being transfected into Cos-1 cells Mouse WT-KDM5B cDNA and human $\triangle \mathrm{JMJC}$-KDM5B, which has the JmjC domain deleted, were also transfected into Cos-1 cells. Cells were then stained for KDM5B and H3K4Me3 and the nucleus along with DAPI. Magnification, $\mathrm{x} 630$, defined by $\mathrm{x} 63$ lens and $\mathrm{x} 10$ eye piece. (B) WT- $K d m 5 B$, $\triangle \mathrm{ARID}-K d m 5 B$ and the vector control were transfected into $293 \mathrm{~T}$ cells before the nuclear proteins were extracted. Demethylase activity was measured using a commercial kit. ${ }^{* *} \mathrm{P}<0.01$ vs. WT-KDM5B. Jmj, Jumonji; ARID, A-T rich interaction domain; KDM5, lysine-specific demethylase 5B; WT, wild-type.

$M D$ simulation reveals conformational changes between wild type KDM5B and DARID-KDM5B. Molecular modelling of WT-KDM5B and $\triangle$ ARID-KDM5B after $100 \mathrm{~ns}$ MD simulation was performed (Fig. $3 \mathrm{~A}$ and B). As with the structural models, the JmjN and JmjC domains are in close proximity to each other in WT-KDM5B, whereas this association appeared to be disturbed in $\triangle$ ARID-KDM5B (Fig. 3A and B). Further comparison of these two models obtained from the last frame of $100 \mathrm{nsec}$ MD simulation revealed that the JmjC domain appeared more compact in the $\triangle$ ARID-KDM5B model compared with that of WT-KDM5B (Fig. 3A and B). This observation was further evidenced by comparing the atomic positional fluctuations of the $\mathrm{C} \alpha$ atoms in WT-KDM5B compared with those in $\triangle$ ARID-KDM5B during the MD simulation (Fig. 3C). Although there were differences in the root mean square fluctuations within the truncated JmjN domain of $\triangle$ ARID-KDM5B, these positional fluctuations were particularly pronounced in the JmjC domain compared with WT-KDM5B (Fig. 3C and D). This suggests that deletion of the 69-DWQPP-73 sequence and the ARID domain resulted in conformational changes in the JmjC domain (residues 453-619) that increased the flexibility of this domain (Fig. 3D). This observation was further supported by 
A

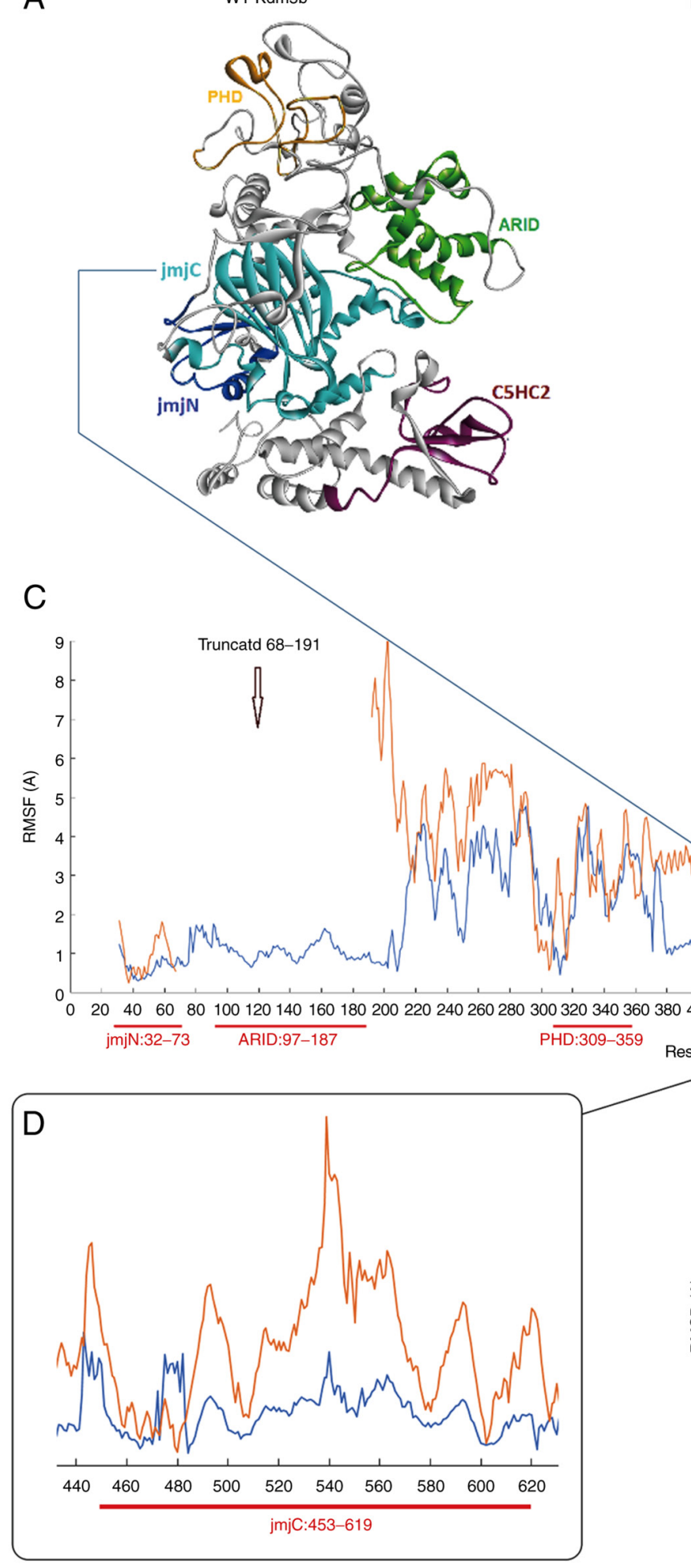

B

$\triangle A R I D K d m 5 b$

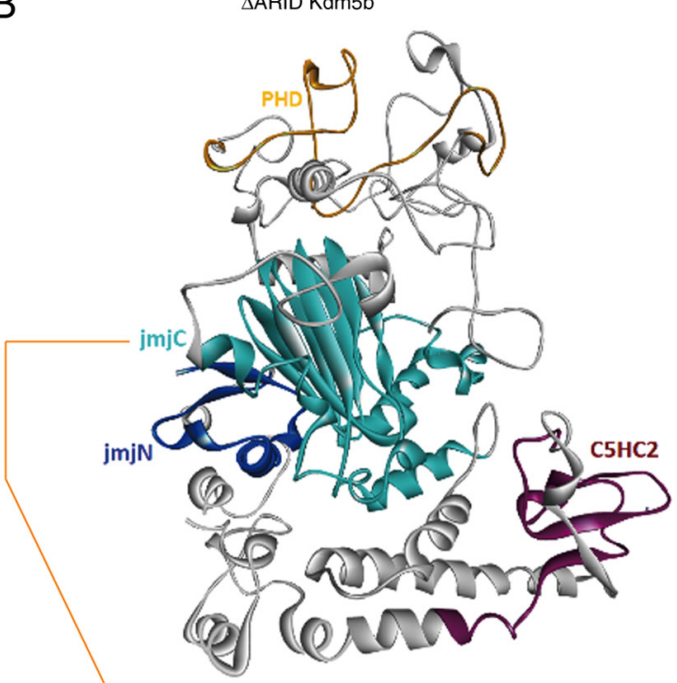

Figure 3. $\triangle$ ARID-KDM5B shows changes in the conformation of the JmjC domain. Molecular models of (A) WT-KDM5B and (B) $\triangle$ ARID-KDM5B obtained from the last frame of the $100 \mathrm{nsec}$ MD simulation. (C) Atomic positional fluctuations, in $\AA$, of $\mathrm{C} \alpha$ atoms in the WT-KDM5B, represented by the blue line, compared with those in the $\triangle$ ARID-KDM5B, represented by the brown line. (D) The residues in the JmjC domain of the $\triangle$ ARID-KDM5B show notably increased degrees of flexibility compared with that in WT-KDM5B. (E) Time dependence of root mean square deviation (RMSD), also in Å, for the backbone atoms relative to the starting structure during $100 \mathrm{nsec}$ MD simulations of both WT-KDM5B (blue) and $\triangle$ ARID-KDM5B (brown). Jmj, Jumonji; ARID, A-T rich interaction domain; KDM5, lysine-specific demethylase 5B; RMSF, root mean square fluctuations; RMSD, root mean square deviation; WT, wild-type; $\mathrm{MD}$, molecular dynamics.

the time dependence of root mean square deviation (RMSD) for the backbone atoms relative to the starting structure during $50 \mathrm{~ns}$ MD simulations in both WT-KDM5B and $\triangle$ ARID-KDM5B (Fig. 3E). The RMSD curves show that both simulations have reached equilibrium after $\sim 30 \mathrm{nsec}$, which was indicated by the relatively stable RMSD values from $30 \mathrm{nsec}$ onwards until the end of the simulations (Fig. 3E). However, the $\triangle$ ARID-KDM5B had a much higher RMSD 
value of of 8-10 $\AA$, compared with 4-5 $\AA$ for WT-KDM5B (Fig. 3E), meaning that $\triangle$ ARID-KDM5B initially had a higher degree of instability but with time reached a stable state. This suggests greater fluctuations and altered dynamics in the protein structure of $\triangle$ ARID-KDM5B.

\section{Discussion}

Both JmjN and JmjC are required for the core demethylase enzymatic activity $(7,8)$. Although separated by other sequences, in the 3-D protein structure these domains lie adjacent to each other (14-18). The present study revealed that the $\triangle$ ARID-KDM5B protein, where the ARID domain and five amino acids from the carboxyl end of the JmjN domain are deleted, has lost its demethylase activity. While previous mutational experiments show that deletion of the JmjN domain results in loss of enzyme activity $(7,8)$, the downstream effects of the partial deletion the JmjN domain as previously seen in the $\triangle$ ARID mouse has not been previously investigated. The splice variant of KDM5B expressed in the $\triangle$ ARID mouse is the only form of KDM5B RNA expressed and produced by using a targeting vector designed to remove exons 2-4 (20). Compared with the embryonic lethal KDM5B KO strains where several strains were identified, only one strain of $\triangle$ ARID mouse could be identified, indicating that the splicing of exon 1 to exon 5 is a rare event (20). Since the last 15 bases in exon 2 translate into the final five amino acid residues (Asp69, Trp70, Gln71, Pro72 and Pro73) of the JmjN domain, these amino acids were deleted from the expressed protein (20). These five amino acids are $100 \%$ conserved between the mouse and human KDM5 families (6) and tend to pack closely with the JmjC domain (14-16), suggesting that the deletion of these amino acids would negatively affect catalytic function.

Previous studies by Horton et al (17) and Johansson et al (14) strongly support the concept that deletion of the ARID and PHD1 domains from KDM5B would not abolish demethylase activity per se. However, the PHD1 domain can be crucial for the recruitment of KDM5A or KDM5B to H3K4me0 (13-15). The PHD1 domain is not deleted in $\triangle$ ARID-KDM5B.

Modelling and subsequent MD simulation of the protein confirms that the JmjN and JmjC regions of the protein are juxtaposed in WT-KDM5B $(14,17)$. In addition, the residues in the truncated JmjN domain of $\triangle$ ARID-KDM5B showed increased degrees of fluctuations compared with WT-KDM5B, suggesting a change in conformation. Changes in the atomic positional fluctuations of the JmjC domain were also observed during the MD simulation, which provides in silico findings that support the experimental observation that $\triangle$ ARID-KDM5B has no demethylase activity. However, deletion of the $\triangle$ ARID domain and five amino acids in the $\mathrm{JmjN}$ domain did not result in differences in the conformation of other domains flanking the JmjC domain, highlighting the importance of the JmjN and JmjC domains in the catalytic activity of KDM5B. Indeed, the fact that the PHD1 domain did not show any significant fluctuations suggests that this demethylase-null KDM5B protein retains its function in the PHD1 domain, including its recruitment to $\mathrm{H} 3 \mathrm{~K} 4 \mathrm{me} 0$ (13). RMSD analysis in the present study suggests that despite the greater fluctuations, $\triangle \mathrm{ARID}-\mathrm{KDM} 5 \mathrm{~B}$ is a stable protein, since both the WT-KDM5B and $\triangle$ ARID-KDM5B systems reached equilibrium after $30 \mathrm{nsec}$ and remained stable for the remaining $70 \mathrm{nsec}$ of the simulation.

To the best of our knowledge, $\triangle$ ARID mice express an experimentally-induced variant of KDM5B (20) that has not been reported to be observed in humans. However, other variants of KDM5B have been reported in patients with intellectual disability (ID) (28). One such variant showing the loss of exon 4, which encodes a part of the ARID domain and leads to an in-frame change, has been identified in identical twins with ID (28). Since sufficient amounts of variant protein could not be isolated, its effect on demethylase activity could not be assayed. Nevertheless, this finding from a patient with ID emphasises the importance of checking brain function in mice expressing $\triangle$ ARID-KDM5B. Indeed, KDM5B is highly expressed in embryonic mouse brain (22), and in the adult brain KDM5B negatively regulates the neurogenesis of neural stem cells (29). In addition, in Drosophila, flies lacking the KDM5 demethylase activity (LID) remain viable and fertile but show behavioural defects (30).

KDM5B was identified as being upregulated in breast and prostate cancer (6-8) and it has been widely studied in these cancers. A KDM5B variant (RBP2-H1), which can be found at lower levels in some normal tissues, such as testis (23), has also been found to be expressed at higher levels in the majority of melanomas (31). This isoform contains additional amino acids (aa238-274) corresponding to exon 6 (31), which is normally absent in the dominant form of KDM5B. In breast cancer, KDM5B is expressed most highly in the estrogen receptor-positive subgroup and is classified as a luminal lineage driving oncogene (32). However, it was also found to be upregulated in other cancers, including bladder, lung, gastric and liver (9,33-35). Evidence that KDM5B can drive cancer cell proliferation comes from previous observations that its levels of expression correlated with poor prognosis and that knocking down its expression resulted in the inhibition of cell proliferation in some cancer cell lines, including colorectal and hepatocellular carcinoma lines (36-38). These data have led to the search for small-molecule weight inhibitors of KDM5B for potential clinical applications $(14,16,39-42)$. The inhibitors that are being developed, with a view to targeting the KDM5 proteins for cancer therapy, are primarily focused on the inhibition of demethylase function $(14,16,39-42)$.

In the present study, the importance of the ARID domain and the five amino acids of the C-terminus of the JmjN domain on the demethylase function were not compared. This should be addressed in a future study. However, results from the present study show that the $\triangle$ ARID-KDM5B is catalytically inactive for the demethylation of methylated $\mathrm{H} 3 \mathrm{~K} 4$, yet mice expressing this mutant remain healthy and fertile (20). By contrast, $K d m 5 b$-knockout is either embryonic lethal (20) or results in neonatal lethality (19). This indicates that although the KDM5B protein serves crucial functions in development, its demethylase activity is dispensable. It is therefore important to evaluate if these functions and the domains responsible for their activity are involved in oncogenesis. The $\triangle$ ARID mouse model could provide a model for investigating this and for addressing other questions, including whether the demethylase-independent effect on mitochondrial function seen in the Drosophila KDM5 analogue LID, is also seen in mammalian KDM5B (30). 


\section{Acknowledgements}

Not applicable.

\section{Funding}

The present study was funded by King's College London, CRUK KHP Centre Grant (grant no. C604/A25135) and the Blood Cancer UK (grant no. 20002). The authors also would like to extend their thanks to the Biotechnology and Biological Sciences Research (grant no. BB/T007737/1) and Medical Research Council (grant no. MR/T015845/1) for funding SJ postdoctoral research associate (PDRA) position within the laboratory of KMR.

\section{Availability of data and materials}

The datasets used and/or analyzed during the current study are available from the corresponding author on reasonable request.

\section{Authors' contributions}

SJ and KMR performed the homology modelling and molecular dynamics stimulation. KMR contributed designing and interpreting the simulation modelling experiments. SC made the constructs and performed the transfection studies, JC and CWES performed the demethylase assays, JB and JTP conceived and designed the study, and interpreted the data. SC, JB and JTP confirmed the authenticity of the data presented in Figs. 1 and 2. JC and CWES confirm the authenticity of the data presented in Fig. 2, SJ and KMR confirm the authenticity of the data presented in Fig. 3. All authors read and approved the final version of this manuscript.

\section{Ethics approval and consent to participate}

Not applicable.

\section{Patient consent for publication}

Not applicable.

\section{Competing interests}

The authors declare that they have no competing interests.

\section{References}

1. Bernstein BE, Kamal M, Lindblad-Toh K, Bekiranov S, Bailey DK, Huebert DJ, McMahon S, Karlsson EK, Kulbokas EJ III, Gingeras TR, et al: Genomic maps and comparative analysis of histone modifications in human and mouse. Cell 120: 169-181, 2005.

2. Cruz C, Della Rosa M, Krueger C, Gao Q, Horkai D, King M, Field L and Houseley J: Tri-methylation of histone H3 lysine 4 facilitates gene expression in ageing cells. Elife 7: e34081, 2018.

3. Roa CR and Dou Y: Hijacked in cancer: The KMT2 (MLL) family of methyltransferases. Nat Rev Cancer 15: 334-346, 2015.

4. Maiques-Diaz A and Somervaille TC: LSD1: Biologic roles and therapeutic targeting. Epigenomics 8: 1103-1016, 2016.

5. Harmeyer KM, Facompre ND, Herlyn M and Basu D: JARID1 histone demethylases: Emerging targets in cancer. Trends Cancer 3: 713-725, 2017.
6. Lu PJ, Sundquist K, Baeckstrom D, Poulsom R, Hanby A, Meier-Ewert S, Jones T, Mitchell M, Pitha-Rowe P, Freemont P and Taylor-Papadimitriou J: A novel gene (PLU-1) containing highly conserved putative DNA/chromatin binding motifs is specifically up-regulated in breast cancer. J Biol Chem 274: 15633-15645, 1999.

7. Yamane K, Tateishi K, Klose RJ, Fang J, Fabrizio LA, Erdjument-Bromage $\mathrm{H}$, Taylor-Papadimitriou J, Tempst $\mathrm{P}$ and Zhang Y: PLU-1 is an H3K4 demethylase involved in transcriptional repression and breast cancer cell proliferation. Mol Cell 25: 801-812, 2007.

8. Xiang Y, Zhu Z, Han G, Ye X, Xu B, Peng Z, Ma Y, Yu Y, Lin H, Chen AP and Chen CD: JARID1B is a histone H3 lysine 4 demethylase up-regulated in prostate cancer. Proc Natl Acad Sci USA 104: 19226-19231, 2007.

9. Xhabija B and Kidder BL: KDM5B is a master regulator of the H3K4-methylome in stem cells, development and cancer. Semin Cancer Biol 57: 79-85, 2019.

10. Jose A, Shenoy GG, Sunil Rodrigues G, Kumar NAN, Munisamy M, Thomas L, Kolesar J, Rai G, Rao PPN and Rao M: Histone demethylase KDM5B as a therapeutic target for cancer therapy. Cancers (Basel) 12: 2121, 2020.

11. Scibetta AG, Santangelo S, Coleman J,Hall D, Chaplin T, Copier J, Catchpole S, Burchell J and Taylor-Papadimitriou J: Functional analysis of the transcription repressor PLU-1/JARID1B. Mol Cell Biol 27: 7220-7235, 2007.

12. Tu S, Teng YC, Yuan C, Wu YT, Chan MY, Cheng AN, Lin PH, Juan LJ and Tsai MD: The ARID domain of H3K4 demethylase RBP2 binds to a DNA CCGCCC motif. Nat Struct Mol Bio 15: 419-421, 2008.

13. Klein BJ, Piao L, Xi Y, Rincon-Arano H, Rothbart SB, Peng D, Wen $\mathrm{H}$, Larson $\mathrm{C}$, Zhang $\mathrm{X}$, Zheng $\mathrm{X}$, et al: The histone-H3K4-specific demethylase KDM5B binds to its substrate and product through distinct PHD fingers. Cell Rep 6: 315-335, 2014.

14. Johansson C, Velupillai S, Tumber A, Szykowska A, Hookway ES, Nowak RP, Strain-Damerell C, Gileadi C, Philpott M, Burgess-Brown N, et al: Structural analysis of human KDM5B guides histone demethylase inhibitor development. Nat Chem Biol 12: 539-545, 2016.

15. Longbotham JE, Chio CM, Dharmarajan V, Trnka MJ, Torres IO, Goswami D, Ruiz K, Burlingame AL, Griffin PR and Fujimori DG: Histone H3 binding to the PHD1 domain of histone demethylase KDM5A enables active site remodeling. Nat Commun 10: 94, 2019.

16. Vinogradova M, Gehling VS, Gustafson A, Arora S, Tindell CA, Wilson C, Williamson KE, Guler GD, Gangurde P, Manieri W, et al: An inhibitor of KDM5 demethylases reduces survival of drug-tolerant cancer cells. Nat Chem Biol 12: 531-538, 2016.

17. Horton JR, Engstrom A, Zoeller EL, Liu X, Shanks JR, Zhang X, Johns MA, Vertino PM, Fu H and Cheng X: Characterization of a linked jumonji domain of the KDM5/JARID1 family of histone H3 lysine 4 demethylases. J Biol Chem 291: 2631-2646, 2016.

18. Torres IO, Kuchenbecker KM, Nnadi CI, Fletterick RJ, Kelly MJ and Fujimori DG: Histone demethylase KDM5A is regulated by its reader domain through a positive-feedback mechanism. Nat Commun 6: 6204, 2015

19. Albert M, Schmitz SU, Kooistra SM, Malatesta M, Morales Torres C, Rekling JC, Johansen JV, Abarrategui I and Helin K: The histone demethylase Jaridlb ensures faithful mouse development by protecting developmental genes from aberrant H3K4me3. PLoS Genet 9: e1003461, 2013.

20. Catchpole S, Spencer-Dene B, Hall D, Santangelo S, Rosewell I, Guenatri M, Beatson R, Scibetta AG, Burchell JM and Taylor-Papadimitriou J: PLU-1/JARID1B/KDM5B is required for embryonic survival and contributes to cell proliferation in the mammary gland and in $\mathrm{ER}^{+}$breast cancer cells. Int J Oncol 38: 1267-1277, 2011

21. Zou MR, Cao J, Liu Z, Huh SJ, Polyak K and Yan Q: Histone demethylase jumonji AT-rich interactive domain 1B (JARID1B) controls mammary gland development by regulating key developmental and lineage specification genes. J Biol Chem 289: 17620-17633, 2014.

22. Madsen B, Spencer-Dene B, Poulsom R, Hall D, Lu PJ, Scott K, Shaw AT, Burchell JM, Freemont P and Taylor-Papadimitriou J: Characterisation and developmental expression of mouse Plu-1, a homologue of a human nuclear protein (PLU-1) which is specifically up-regulated in breast cancer. Mech Dev 119 (Suppl 1): S239-S246, 2002. 
23. Barrett A, Madsen B, Copier J, Lu PJ, Cooper L, Scibetta AG, Burchell $\mathrm{J}$ and Taylor-Papadimitriou J: PLU-1 nuclear protein, which is upregulated in breast cancer, shows restricted expression in normal human adult tissues: A new cancer/testis antigen? Int J Cancer 101: 581-586, 2002.

24. Arnold K, Bordoli L,Kopp J and Schwede T: The SWISS-MODEL workspace: A web-based environment for protein structure homology modelling. Bioinformatics 22: 195-201, 2006.

25. Schwede T, Kopp J, Guex N and Peitsch M: Swiss-Model: An automated protein homology-modeling server. Nucleic Acids Res 31: 3381-3385, 2003.

26. Case DA, Cheatham TE III, Darden T, Gohlke H, Luo R, Merz KM Jr, Onufriev A, Simmerling C, Wang B and Woods RJ: The Amber biomolecular simulation programs. J Comput Chem 26: 1668-1688, 2005

27. Darden T, York D and Pedersen L: Particle mesh Ewald: An N $\log (\mathrm{N})$ method for Ewald sums in large systems. J Chem Phys 98 10089-10092, 1993

28. Lebrun N, Mehler-Jacob C, Poirier K, Zordan C, Lacombe D, Carion N, Billuart P and Bienvenu T: Novel KDM5B splice variants identified in patients with developmental disorders: Functional consequences. Gene 679: 305-313, 2018.

29. Zhou Q, Obana EA, Radomski KL, Sukumar G, Wynder C Dalgard CL and Doughty ML: Inhibition of the histone demethylase Kdm5b promotes neurogenesis and derepresses Reln (reelin) in neural stem cells from the adult subventricular zone of mice. Mol Biol Cell 27: 627-639, 2016.

30. Zamurrad S, Hatch HAM, Drelon C, Belalcazar HM and Secombe J: A Drosophila model of intellectual disability caused by mutations in the histone demethylase KDM5. Cell Rep 22: 2359-2369, 2018

31. Kuźbicki Ł, Lange D, Stanek-Widera A and Chwirot BW: Prognostic significance of RBP2-H1 variant of JARID1B in melanoma. BMC Cancer 17: 854, 2017.

32. Yamamoto S, Wu Z, Russnes HG, Takagi S, Peluffo G, Vaske C, Zhao X, Moen Vollan HK, Maruyama R, Ekram MB, et al: JARID1B is a luminal lineage-driving oncogene in breast cancer. Cancer Cell 25: 762-777, 2014.

33. Hayami S, Yoshimatsu M, Veerakumarasivam A, Unoki M, Iwai Y, Tsunoda T, Field HI, Kelly JD, Neal DE, Yamaue H, et al: Overexpression of the JmjC histone demethylase KDM5B in human carcinogenesis: Involvement in the proliferation of cancer cells through the E2F/RB pathway. Mol Cancer 9: 59, 2010.
34. Shen X, Zhuang Z, Zhang Y, Chen Z, Shen L, Pu W, Chen L and $\mathrm{Xu} Z \mathrm{Z}$ : JARID1B modulates lung cancer cell proliferation and invasion by regulating p53 expression. Tumour Biol 36: 7133-7142, 2015

35. Tang B, Qi G, Tang F, Yuan S, Wang Z, Liang X, Li B, Yu S, Liu J, Huang Q, et al: JARID1B promotes metastasis and epithelial-mesenchymal transition via PTEN/AKT signaling in hepatocellular carcinoma cells. Oncotarget 6: 12723-12739, 2015.

36. Yan G, Li S, Yue M, Li C and Kang Z: Lysine demethylase 5B suppresses $\mathrm{CC}$ chemokine ligand 14 to promote progression of colorectal cancer through the Wnt/ $\beta$-catenin pathway. Life Sci 264: 118726, 2021.

37. Shigekawa Y,Hayami S, Ueno M, Miyamoto A, Suzaki N, Kawai M, Hirono S, Okada KI, Hamamoto R and Yamaue H: Overexpression of KDM5B/JARID1B is associated with poor prognosis in hepatocellular carcinoma. Oncotarget 9: 34320-34335, 2018.

38. Montano MM, Yeh IJ, Chen Y, Hernandez C, Kiselar JG, de la Fuente M, Lawes AM, Nieman MT, Kiser PD, Jacobberger J, et al: Inhibition of the histone demethylase, KDM5B, directly induces re-expression of tumor suppressor protein HEXIM1 in cancer cells. Breast Cancer Res 21: 138, 2019.

39. Sayegh J, Cao J, Zou MR, Morales A, Blair LP, Norcia M, Hoyer D, Tackett AJ, Merkel JS and Yan Q: Identification of small molecule inhibitors of Jumonji AT-rich interactive domain 1B (JARID1B) histone demethylase by a sensitive high throughput screen. J Biol Chem 288: 9408-9417, 2013.

40. Tumber A, Nuzzi A, Hookway ES, Hatch SB, Velupillai S, Johansson C, Kawamura A, Savitsky P, Yapp C, Szykowska A, etal: Potent and selective KDM5 inhibitor stops cellular demethylation of H3K4me3 at transcription start sites and proliferation of MM1S myeloma cells. Cell Chem Biol 24: 371-380, 2017.

41. Højfeldt JW, Agger K and Helin K: Histone lysine demethylases as targets for anticancer therapy. Nat Rev Drug Discov 12: 917-930, 2013.

42. Kristensen LH, Nielsen AL, Helgstrand C, Lees M, Cloos P, Kastrup JS, Helin K, Olsen L and Gajhede M: Studies of H3K4me3 demethylation by KDM5B/Jarid1B/PLU1 reveals strong substrate recognition in vitro and identifies 2,4-pyridine-dicarboxylic acid as an in vitro and in cell inhibitor. FEBS J 279: 1905-1914, 2012.

This work is licensed under a Creative Commons Attribution-NonCommercial-NoDerivatives 4.0 International (CC BY-NC-ND 4.0) License. 